2017-04

\title{
Analytical investigation of hydrodynamic performance of a dual pontoon WEC-type breakwater
}

Ning, D-Z

http://hdl.handle.net/10026.1/9636

10.1016/j.apor.2017.03.012

Applied Ocean Research

Elsevier BV

All content in PEARL is protected by copyright law. Author manuscripts are made available in accordance with publisher policies. Please cite only the published version using the details provided on the item record or document. In the absence of an open licence (e.g. Creative Commons), permissions for further reuse of content should be sought from the publisher or author. 


\title{
WEC-type breakwater
}

\author{
De-Zhi Ning ${ }^{* 1}$, Xuan-Lie Zhao ${ }^{1}$, Ming Zhao ${ }^{1,2}$, Martyn Hann ${ }^{3}$, Hai-Gui Kang ${ }^{1}$ \\ ${ }^{1}$ State Key Laboratory of Coastal and Offshore Engineering, Dalian University of Technology, \\ Dalian, 116024, China \\ ${ }^{2}$ School of Computing, Engineering and Mathematics, University of Western Sydney, Locked Bay 1797, Penrith, \\ NSW 2751, Australia \\ ${ }^{3}$ School of Marine Science and Engineering, Plymouth University, Plymouth, PL4 8AA,UK
}

\section{Abstract}

Based on the linear potential flow theory and matching eigen-function expansion technique, an analytical model is developed to investigate the hydrodynamics of two-dimensional dual-pontoon floating breakwaters that also work as oscillating buoy wave energy converters (referred to as the integrated system hereafter). The pontoons are constrained to heave motion independently and the linear power take-off damping is used to calculate the absorbed power. The proposed model is verified by using the energy conservation principle. The effects of the geometrical parameters on the hydrodynamic properties of the integrated system, including the reflection and transmission coefficients and CWR (capture width ratio, which is defined as the ratio of absorbed wave power to the incident wave power in the device width). It is found that the natural frequency of the heave motion and the spacing of the two pontoons are the critical factors affecting the performance of the integrated system. The comparison between the results of the dual-pontoon breakwater and those of the single-pontoon breakwater shows that the effective frequency range (for condition of transmission coefficient $K_{\mathrm{T}}<0.5$ and the total capture width ratio $\eta_{\text {total }}>20 \%$ ) of the dual-pontoon system is broader than that of the single-pontoon system with the same total volume.

Key words: linear potential flow theory; floating breakwaters; wave energy extraction; effective frequency range.

\section{Introduction}

Extracting energy from ocean waves has become an important research focusing in ocean

\footnotetext{
*Corresponding author. Email: dzning@dlut.edu.cn
} 
engineering in recent years. To date, a wide variety of wave energy converters (WECs) have been developed, such as oscillating water column (OWC), oscillating buoy and overtopping wave energy converters (Falcão, 2010). However, the high construction cost of these energy conversion devices is still a big challenge (Ferro, 2006). Therefore, reducing the cost of wave energy devices through the improved design has become utmost important.

One solution to reduce the cost is the concept of embedding WECs into other offshore structures (Vicinanza et al., 2014). Combining the wave energy devices with breakwaters has drawn increasing attention during the past years. Takahashi et al. (1992), Boccotti (2007) and Arena et al. (2013) proposed the concept of building wave energy devices into caisson breakwaters. The wave energy devices were integrated into the pile-supported breakwater, the rubble mound breakwaters and the submerged plate-type breakwater by He and Huang (2014), Vicinanza et al. (2014) and Orer and Ozdamar (2007), respectively. It is understood that the floating breakwaters are preferable due to their relatively low costs, independence on subsea geological conditions, low environmental impact, aesthetic considerations and flexibility (McCartney, 1985). He et al. (2012, 2013), Michailides and Angelides (2012), Ning et al. (2016), Martinelli et al. (2016) and Chen et al. (2016) investigated the performance of hybrid systems consisting of a floating breakwater and a wave energy extraction device. In addition, some research has been conducted to study the coastal protection of wave farms (Zanuttigh and Angelelli, 2013; Mendoza et al., 2014). From the literature, the advantages of the integrated systems can be concluded as follows: (1) the cost sharing between wave energy devices and breakwaters can be achieved; (2) the additional ocean space is unneeded for the wave energy device; (3) the multi-purpose use of WECs may be achieved.

This study follows the study by Ning et al. (2016). They conducted laboratory experiments to evaluate the performance of an integrated system consisting of an oscillating buoy WECs and a pile-restrained floating breakwater. In this study, an analytical method is developed to calculate the performance of an integrated system, which allows the parametric studies in a wide range of wave and structural parameters. The transmission and reflection coefficients are the important factors to evaluate the performance of a breakwater and the capture width ratio is often used to quantify the performance of the WECs. It is understood that breakwaters are often considered as operating satisfactory when $K_{\mathrm{T}}<0.5$ ( $K_{\mathrm{T}}$ denotes the transmission coefficient) and the effective capture width ratio CWR for a wave energy converter shall be greater than 20\% (Koutandos et al., 2005; Babarit et 
al., 2012; Ning et al., 2016). In this paper, the frequency range corresponding to $K_{\mathrm{T}}<0.5$ and $\mathrm{CWR}>$ $20 \%$ is named as effective frequency bandwidth. For a system with a single pontoon, the qualified transmission coefficient and the effective CWR can be achieved only for a narrow frequency range (Ning et al. (2016)). Additionally, the theoretical maximum energy conversion efficiency is only 50\% and the effective frequency range of energy conversion is narrow for a two-dimensional symmetrical device with heave motion (Falnes, 2002; Arena et al., 2013). The present study aims at broadening the effective frequency bandwidth of the integrated systems by introducing an improved arrangement of dual-pontoon breakwaters. A power take-off (PTO) system is installed on each pontoon to harvest the energy of its heave motion. Two pontoons are arranged in tandem and work independently. The schematic sketch of the improved arrangement is shown in Fig. 1. From the point view of the engineering costs, the total volume of the two breakwaters shall be smaller than that of the case with a single pontoon.

The hydrodynamics of offshore structures consisting of dual pontoons have been studied by many researchers using analytical (Liu and Li, 2014; Zheng and Zhang, 2016), numerical (Weng and Chou, 2007; Williams and Abul-Azm, 1997; Williams et al., 2000) and experimental methods (Koutandos et al., 2005). For structures with regular shapes, analytical methods with high computational efficiency are often used to predict the wave-structure interaction (Li and Teng, 2015). In this study, the analytical method based on the linear potential theory is used to calculate the diffraction and radiation problems of the two-pontoon system. The exciting wave force and hydrodynamic coefficients in the heave mode are computed based on the analytical model for 2-D wave-structure interaction developed by Zheng and Zhang (2016). The reflection and transmission coefficients and the CWR are calculated for a wide parametric range. The rest of the paper is organized as follows. In Section 2, the formulas are described. In Section 3, the validation, the results and the discussions are presented. In Section 4, the conclusions are given.

\section{Analytical formula}

As shown in Fig. 1, a breakwater comprises of dual floating pontoons that are installed in the water with uniform depth $h$. The breadths of the pontoon 1 and pontoon 2 are defined as $a_{1}$ and $a_{2}$, the drafts $d_{1}$ and $d_{2}$, respectively, and the spacing between the two pontoons is $D$. To study the 
interaction between the waves and the floating breakwater, a two-dimensional Cartesian coordinate $(O-x z)$ system is employed with its origin located on the still water surface. Correspondingly, the mass and the stiffness of the $n$th pontoon pontoon in the heave mode can be expressed as $M_{n}$ (= $\left.\rho a_{n} d_{n}\right)$ and $K_{n}\left(=\rho g a_{n}\right)$, respectively, where $\rho$ is the water density, $g$ the gravitational acceleration and $n=1$ or 2 . The structures are subjected to a train of regular waves travelling in the positive $x$-direction and are assumed to respond only in the heave mode only.

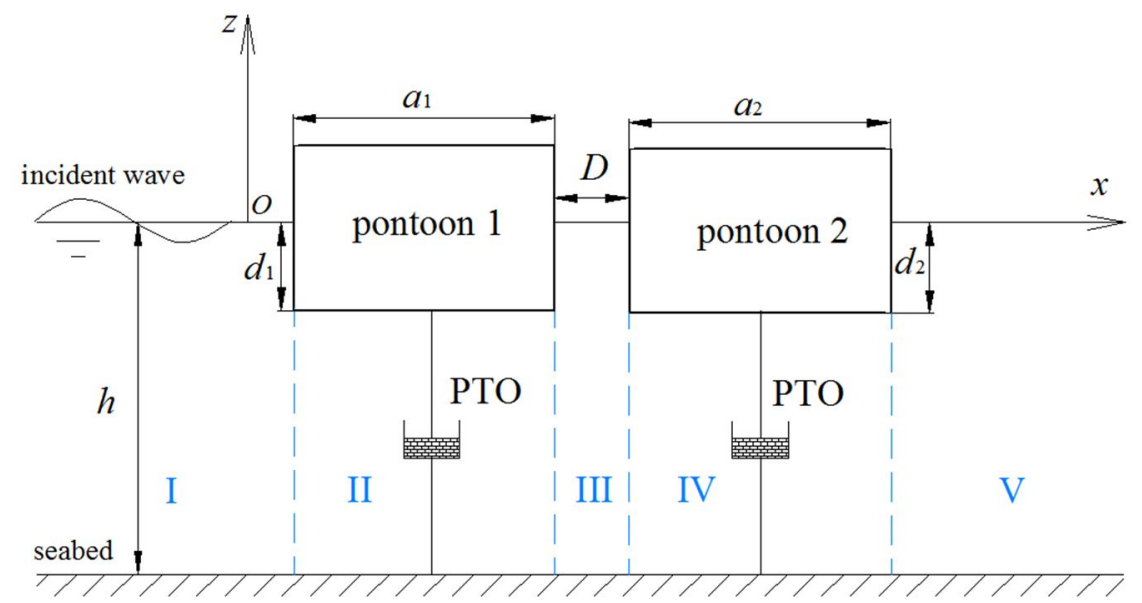

Figure 1 Sketch of the floating structures with the PTO systems

As indicated in Fig. 1, the fluid domain is divided into five subdomains I, II, III, IV and V. The fluid motion in the whole domain can be described by the velocity potential

$$
\phi(x, z, t)=\operatorname{Re}\left[\Phi(x, z) e^{-\mathrm{i} \omega t}\right]
$$

where $t$ is the time, $\mathrm{i}=\sqrt{-1}, \omega$ the angular frequency, Re denotes the real part of a complex, $\Phi$ is a complex velocity potential that satisfies the Laplace equation:

$$
\frac{\partial^{2} \Phi}{\partial x^{2}}+\frac{\partial^{2} \Phi}{\partial z^{2}}=0
$$

The velocity potential $\Phi$ can be divided into three components as:

$$
\Phi=\Phi_{\mathrm{I}}+\Phi_{\mathrm{D}}+\sum_{n=1}^{2} \Phi_{\mathrm{R}, n}
$$

where $\Phi_{\mathrm{I}}$ is the incident potential, $\Phi_{\mathrm{D}}$ the diffraction potential and $\Phi_{\mathrm{R}, n}$ the radiation potential due to the heave motion of the $n$th structure. The velocity potential for the incident waves can be written as 
109 where $A$ is the wave amplitude, $k$ the wave number, which satisfies the dispersion relation, i.e., $\omega^{2}=$ $110 \quad g k \tanh (k h)$.

111 For the diffraction problem, the governing equation is Laplace equation and the boundary 112 conditions can be written as follows:

$$
\left\{\begin{array}{l}
\frac{\partial \Phi_{\mathrm{D}}}{\partial z}-\frac{\omega^{2}}{g} \Phi_{\mathrm{D}}=0 \quad\left(z=0, x<x_{1,1} \text { or } x_{\mathrm{r}, 1}<x<x_{1,2} \text { or } x>x_{\mathrm{r}, 2}\right) \\
\frac{\partial \Phi_{\mathrm{D}}}{\partial z}=0 \quad(z=-h) \\
\frac{\partial \Phi_{\mathrm{D}}}{\partial z}=-\frac{\partial \Phi_{\mathrm{I}}}{\partial z} \quad\left(z=-d_{n}, x_{1, n}<x<x_{\mathrm{r}, n}, n=1,2\right) \\
\frac{\partial \Phi_{\mathrm{D}}}{\partial x}=-\frac{\partial \Phi_{\mathrm{I}}}{\partial x} \quad\left(-d_{n}<z<0, x=x_{\mathrm{r}, n} \text { or } x=x_{1, n}, n=1,2\right) \\
\Phi_{\mathrm{D}} \text { outgoing: finite value, }|x| \rightarrow \infty
\end{array}\right.
$$

114 where $x_{1, n}$ denotes the coordinate of the left edge of the $n$th structure and $x_{\mathrm{r}, n}$ denotes the coordinate of 115 the right edge of the $n$th structure.

The radiation potential due to the heave motion of the $n$th pontoon with an amplitude $A_{\mathrm{R}, n}$ and an angular frequency $\omega$ can be written as

$$
\Phi_{\mathrm{R}, n}=-\mathrm{i} \omega A_{\mathrm{R}, n} \varphi_{\mathrm{R}, n}(x, z)
$$

119 The complex spatial velocity potential $\varphi_{\mathrm{R}, n}$ satisfies the Laplace equation and its boundary 120 conditions can be written as follows:

$$
\left\{\begin{array}{l}
\frac{\partial \varphi_{\mathrm{R}, n}}{\partial z}-\frac{\omega^{2}}{g} \varphi_{\mathrm{R}, n}=0 \quad\left(z=0, \quad x<x_{1,1} \text { or } x_{\mathrm{r}, 1}<x<x_{1,2} \text { or } x>x_{\mathrm{r}, 2}\right) \\
\frac{\partial \varphi_{\mathrm{R}, n}}{\partial z}=0 \quad(z=-h) \\
\frac{\partial \varphi_{\mathrm{R}, n}}{\partial z}=\delta_{m, n} \quad\left(z=-d_{m}, x_{1, m}<x<x_{\mathrm{r}, m}, m=1,2\right) \\
\frac{\partial \varphi_{\mathrm{R}, n}}{\partial x}=0 \quad\left(-d_{m}<z<0, x=x_{\mathrm{r}, m} \text { or } x=x_{1, m}, m=1,2\right) \\
\varphi_{\mathrm{R}, n} \text { outgoing: finite value, }|x| \rightarrow \infty
\end{array}\right.
$$


where $\delta_{m, n}$ is the Kronecker delta.

The analytical expressions of the diffraction and radiation potentials (including the evanescent modes) in each domain can be obtained based on the method by Zheng and Zhang (2016). The equation sets can be formed by substituting the diffraction potentials into Eq. (5) and radiation potentials into Eq. (7) and using the orthogonality of the vertical eigen-function. Then the unknown coefficients of the diffraction and radiation potentials can be obtained. The potential in each domain can be further determined. Then the vertical exciting force $F_{z, n}$ on the $n$th structure can be calculated by

$$
F_{z, n}=-\mathrm{i} \omega \rho \int_{S_{n}}\left(\Phi_{\mathrm{I}}+\Phi_{\mathrm{D}}\right) n_{z} \mathrm{~d} s
$$

where $S_{n}$ is the bottom surface of the $n$th structure and $n_{z}$ is the unit normal vector in the negative $z$-direction.

The added mass $\mu_{n}^{m}$ and radiation damping $\lambda_{n}^{m}$ on the $n$th structure in the heave motion subject to a unit forced motion of the $m$ th structure can be written as:

$$
\begin{gathered}
\mu_{n}^{m}=-\rho \int_{S_{n}} \operatorname{Re}\left[\varphi_{\mathrm{R}, m}\right] n_{z} \mathrm{~d} s \\
\lambda_{n}^{m}=-\rho \omega \int_{S_{n}} \operatorname{Im}\left[\varphi_{\mathrm{R}, m}\right] n_{z} \mathrm{~d} s
\end{gathered}
$$

where Im denotes the imaginary part of a complex and $m=1,2$.

Then the equation of motion can be written as:

$$
\left(-\omega^{2}(\mathbf{M}+\boldsymbol{\mu})-\mathrm{i} \omega\left(\boldsymbol{\lambda}+\boldsymbol{\lambda}_{\text {PTO }}\right)+\mathbf{K}\right) \mathbf{A}_{\mathbf{R}}=\mathbf{F}_{\mathbf{z}}
$$

where $\mathbf{M}$ and $\mathbf{K}$ are the mass and stiffness matrices of the structures, respectively; $\boldsymbol{\mu}$ and $\boldsymbol{\lambda}$ are the added mass and wave damping matrices of the structures, respectively; $\boldsymbol{\lambda}_{\mathbf{P T O}}$ is the PTO damping matrix imposed on the structures. $\mathbf{A}_{\mathbf{R}}$ and $\mathbf{F}_{\mathbf{z}}$ denote heave response motion vector and heave excitation force vector, respectively.

The motion equation can be re-written as:

$$
\left\{-\omega^{2}\left(\left[\begin{array}{cc}
M_{1} & 0 \\
0 & M_{2}
\end{array}\right]+\left[\begin{array}{ll}
\mu_{1}^{1} & \mu_{1}^{2} \\
\mu_{2}^{1} & \mu_{2}^{2}
\end{array}\right]\right)-\mathrm{i} \omega\left(\left[\begin{array}{cc}
\lambda_{1}^{1} & \lambda_{1}^{2} \\
\lambda_{2}^{1} & \lambda_{2}^{2}
\end{array}\right]+\left[\begin{array}{cc}
\lambda_{\mathrm{PTO}}[1,1] & 0 \\
0 & \lambda_{\mathrm{PTO}, 2}[2,2]
\end{array}\right]\right)+\left[\begin{array}{cc}
K_{1} & 0 \\
0 & K_{2}
\end{array}\right]\right\}\left(\begin{array}{l}
A_{R, 1} \\
A_{R, 2}
\end{array}\right)=\left(\begin{array}{l}
F_{z, 1} \\
F_{z, 2}
\end{array}\right)
$$

$$
\text { Note that, for a dual pontoon WEC-type floating breakwater, the nonzero elements of } \lambda_{\text {PTo }} \text { are }
$$


$\lambda_{\mathrm{PTO}}[1,1]$ and $\lambda_{\mathrm{PTO}}[2,2]$, which represent the PTO damping imposed on the first and second pontoon, respectively. In the present study, $\lambda_{\mathrm{PTO}}[n, n]$ equals to the optimal PTO damping for an isolated single

151 device, which can be expressed as $\lambda_{\text {PTO }}[n, n]=\sqrt{\left(K_{n} / \omega-\omega\left(M_{n}+\mu_{n}\right)\right)^{2}+\lambda_{n}{ }^{2}}$, where $\mu_{n}$ and $\lambda_{n}$ 152 represent the added mass and damping coefficient of the $n$th structure in the isolated case (Falnes, 153 2002; Wolgamot et al., 2016).

154 The power $P_{n}$ produced by the $n$th structure can be calculated by:

$$
P_{n}=\frac{1}{2} \omega^{2} \lambda_{\text {PTO }}[n, n]\left|A_{R, n}\right|^{2}
$$

Then the total power absorbed is as follows:

$$
P_{\text {total }}=\sum_{n=1}^{2} P_{n}
$$

The incident wave power can be calculated as follows

$$
P_{\text {incident }}=\frac{1}{4} \frac{\rho g A^{2} \omega}{k}\left(1+\frac{2 h k}{\sinh 2 h k}\right)
$$

The CWR is an important indicator to evaluate the hydrodynamic efficiency of WECs (Babarit, 2015). The CWR $\left(\eta_{n}\right)$ of the $n$th structure can be calculated as $\eta_{n}=P_{n} / P_{\text {incident }}$ and the total CWR as $\eta_{\text {total }}=P_{\text {total }} / P_{\text {incident }}$.

The performance of a breakwater can be evaluated by the reflection coefficients $K_{\mathrm{R}}$ and transmission coefficients $K_{\mathrm{T}}$ :

$$
\begin{aligned}
& K_{R}=\left|\frac{\Phi_{\mathrm{D}}-\mathrm{i} \omega \sum_{n=1}^{2} A_{\mathrm{R}, n} \varphi_{\mathrm{R}, n}}{\Phi_{\mathrm{I}}}\right| \\
& K_{T}=\left|\frac{\Phi_{\mathrm{I}}+\Phi_{\mathrm{D}}-\mathrm{i} \omega \sum_{n=1}^{2} A_{\mathrm{R}, n} \varphi_{\mathrm{R}, n}}{\Phi_{\mathrm{I}}}\right|
\end{aligned}
$$

\section{Results and discussions}

\subsection{Validation}

The present model is validated by using the energy conservation relationship of $K_{\mathrm{R}}{ }^{2}+K_{\mathrm{T}}{ }^{2}+\eta_{\text {total }}$ 
$170=1$. Fig. 2 shows the results of the reflection coefficient $K_{\mathrm{R}}$, the transmission coefficient $K_{\mathrm{T}}$, the total $171 \mathrm{CWR} \eta_{\text {total }}$ and the $K_{\mathrm{R}}{ }^{2}+K_{\mathrm{T}}{ }^{2}+\eta_{\text {total }}$ for geometrical parameters of $a_{1}=a_{2}=6 \mathrm{~m}, d_{1}=d_{2}=1.25 \mathrm{~m}, D$ $172=2 \mathrm{~m}$ and $h=10 \mathrm{~m}$. The nonzero elements of the PTO damping matrix are chosen as $\lambda_{\text {PTO }}[1,1]$ and $173 \lambda_{\text {РTO }}[2,2]$. It can be seen that the relation of $K_{\mathrm{R}}{ }^{2}+K_{\mathrm{T}}{ }^{2}+\eta_{\text {total }}=1$ is satisfied perfectly, which validates the present analytical model.

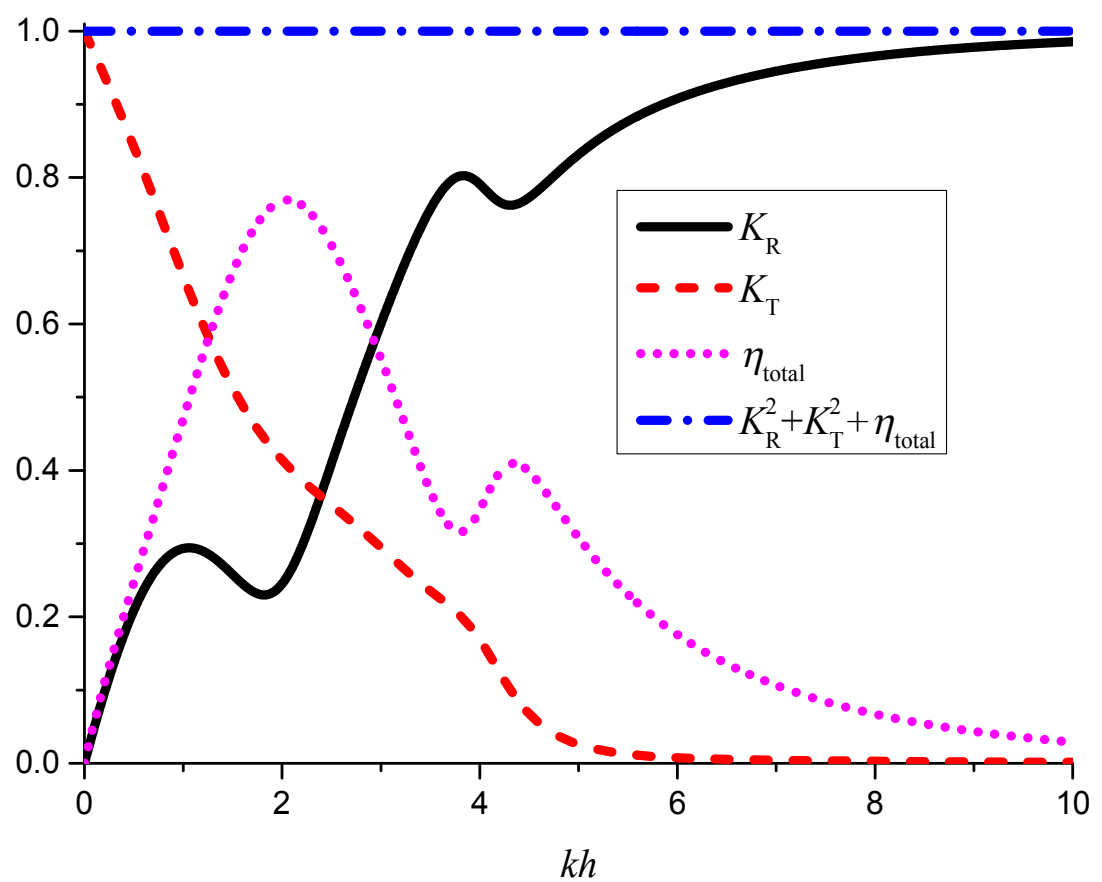
Figure 2 Variations of reflection coefficient $K_{\mathrm{R}}$, transmission coefficient $K_{\mathrm{T}}$, the totoal CWR $\eta_{\text {total }}$ and $K_{\mathrm{R}}^{2}+K_{\mathrm{T}}^{2}+\eta_{\text {total }} v s$ the dimensionless wavenumber $k h$.

\subsection{Parametric study}

The performance of an integrated system combining a dual-pontoon floating breakwater and dual wave energy devices relies on several parameters, including the breadths, drafts, and spacing of the pontoons $\left(a_{n}, d_{n}\right.$ and $\left.D\right)$. A parametric study is conducted to investigate the sensitivity of the performance of an integrated system to various wave and geometrical parameters. The difference between the system with dual pontoons and that with a single pontoon are compared in Section 3.2.3.

\subsubsection{Effect of the structure breadths $\left(a_{1}\right.$ and $\left.a_{2}\right)$}

A breakwater with two identical pontoons is considered in Sections 3.2.1, 3.2.2 and 3.2.3. Firstly, the effect of the structure breadths is investigated. Figs. 3(a-c) show the variation of the reflection coefficient $K_{\mathrm{R}}$, the transmission coefficient $K_{\mathrm{T}}$ and the total CWR $\eta_{\text {total }}$ against the 
189 dimensionless wavenumber $k h$ for three breadths of $a_{1} / h=a_{2} / h=0.4,0.6$ and 0.8 . The other 190 geometrical parameters are kept constant as $d_{1} / h=d_{2} / h=0.125, D / h=0.2$.

191 In each of Fig. 3 (a) - (c), the general trends for all three curves are the same. The reflection 192 coefficient increases with increasing $a_{1} / h$. In particular, it is noted that the reflection coefficient 193 exhibits small oscillations at some critical wave numbers among the generally upward curve and the 194 wave numbers where the minimum and maximum values of $K_{\mathrm{R}}$ occur shift to the lower frequency region with the increase of the structure breadth. This may be due to the interference by the strong reflection. Similarly, oscillations can also be found for the curves of total CWR. However, for the transmission coefficient, the oscillation phenomenon is weak. A similar phenomenon was found by 198 Garnaud and Mei (2009), who adopted the analytical multiple scales method under the framework of 199 linear potential flow theory. The effective frequency ranges of different structure breadths are slightly different from each other. The effective frequency ranges for $a_{1} / h=a_{2} / h=0.4,0.6$ and 0.8 are $2.04<$ $k h<6.25,1.55<k h<5.75$ and $1.26<k h<5.37$, resulting in bandwidths of 4.21, 4.20 and 4.11, respectively.
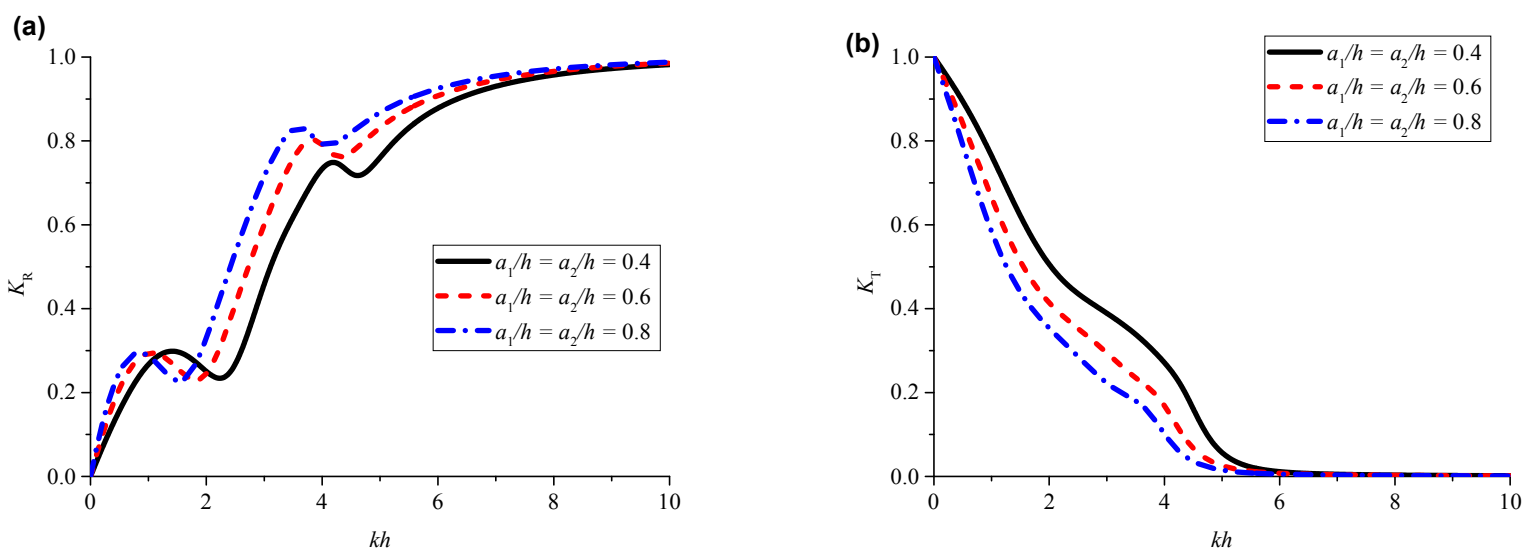

(c)

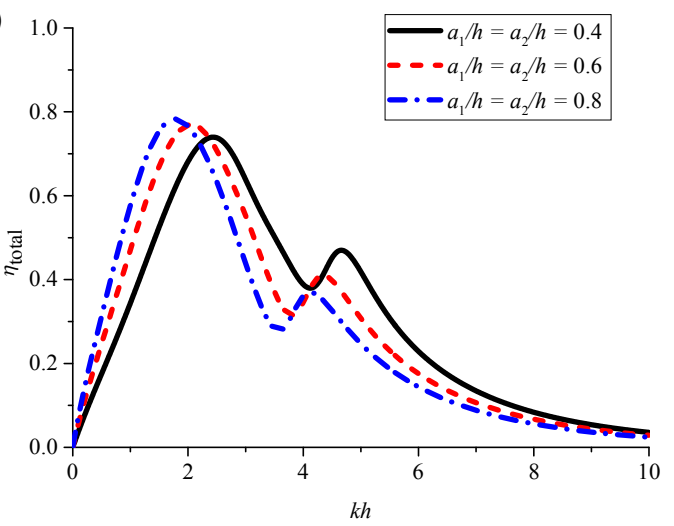

Figure 3 Variations of reflection coefficient $K_{\mathrm{R}}$, transmission coefficient $K_{\mathrm{T}}$ and CWR $\eta_{\text {total }} v s$ the 
206 dimensionless wavenumber $k h$ for cases with different structure breadths $\left(d_{1} / h=d_{2} / h=0.125, D / h=\right.$ 207 0.2).

\section{$208 \quad 3.2 .2$ Effect of the structure drafts $\left(\boldsymbol{d}_{\mathbf{1}}\right.$ and $\left.\boldsymbol{d}_{\mathbf{2}}\right)$}

209 Figs. 4 (a-c) present the influence of the drafts on the performance of the integrated system. 210 Results are shown for three cases with drafts of $d_{1} / h=d_{2} / h=0.08,0.125$ and 0.15 . The other 211 parameters are kept constant as $a_{1} / h=a_{2} / h=0.6, D / h=0.2$. All the reflection coefficients increases 212 with increasing $k h$ with some oscillations. The critical wave number corresponding to the maximum 213 values of $K_{\mathrm{R}}$ shifts towards the lower frequency with the increase of the draft. As expected, the 214 structures with larger draft provide the more effective wave barriers, and the effect of the draft on the $215 K_{\mathrm{R}}$ is more obvious for $2<k h<7$ than the other $k h$. However, it appears that the effect of the draft on $216 \eta_{\text {total }}$ is in the apparently opposite trend to that on $K_{\mathrm{R}}$ as $k h>2.5$. In addition, the wave numbers 217 where $\eta_{\text {total }}$ reaches their maximum correspond to those where $K_{\mathrm{R}}$ values reaches its minimum. The 218 effective frequency range and the peak value of $\eta_{\text {total }}$ decreases with the increase of the draft ratio. 219 The effective frequency ranges for $d_{1} / h=d_{2} / h=0.08,0.125$ and 0.15 are $1.65<k h<7.93,1.55<k h$ $220<5.76$ and $1.50<k h<5.00$, and the corresponding effective bandwidths are 6.28, 4.21 and 3.50, 221 respectively. 
(a)

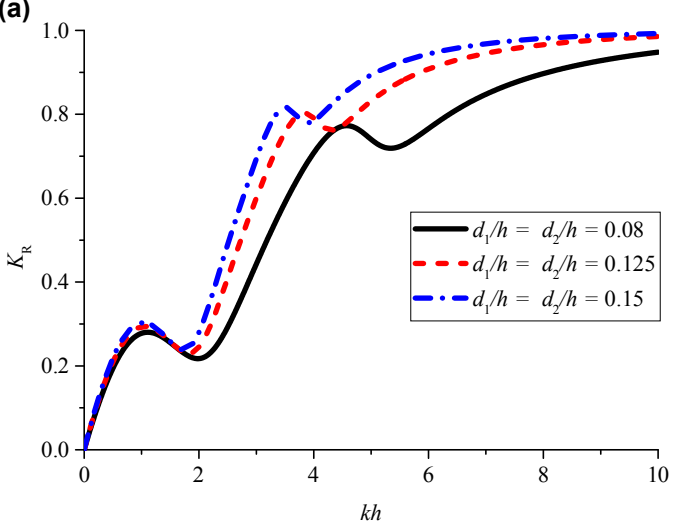

(b)

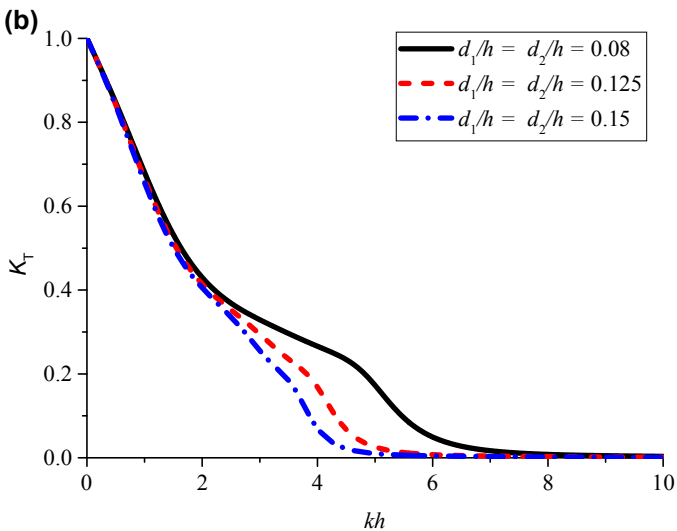

(c)

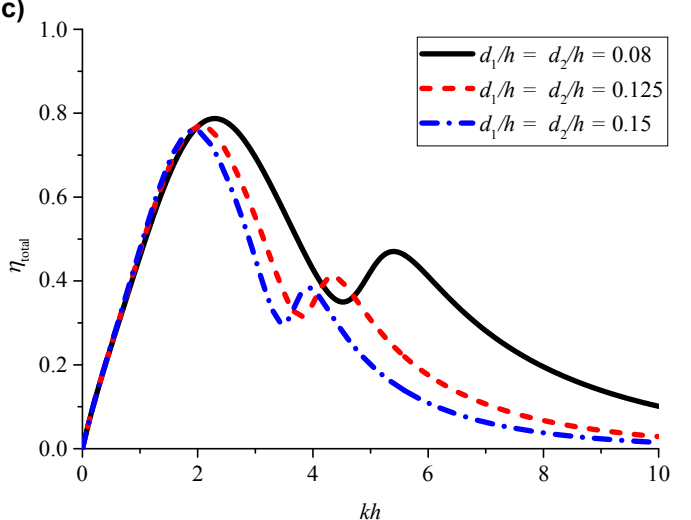

Figure 4 Variations of the reflection coefficient $K_{\mathrm{R}}$, transmission coefficient $K_{\mathrm{T}}$ and total capture width ratio $\eta_{\text {total }} v s$ the dimensionless wavenumber $k h$ for cases with different structure drafts $\left(a_{1} / h=\right.$ $\left.a_{2} / h=0.6, D / h=0.2\right)$.

It can be understood that, for a two-dimensional pontoon, the natural frequency of the heave mode decreases with the increase of the structure breadth or draft, which can be identified by Figs. 3 and 4. By combing the results in Figs. 3 and 4, it can be seen that breakwater performance of the system becomes better and the performance of the energy conversion becomes worse with the decrease of the natural frequency of the heave mode.

\subsubsection{Effect of the pontoon spacing $(D)$}

The effect of pontoon spacing on the reflection coefficient $K_{\mathrm{R}}$, transmission coefficient $K_{\mathrm{T}}$ and total CWR $\eta_{\text {total }}$ is shown in Figs. 5(a-c) for four pontoons of $D / h=0.2,0.4,0.6$ and 1.0. Other geometrical parameters are kept constant as $a_{1} / h=a_{2} / h=0.6$ and $d_{1} / h=d_{2} / h=0.125$. For comparisons, the results of a single pontoon of $a / h=0.6$ and $d / h=0.25$ (referred to be single pontoon 1 , where $a$ is the breadth of the pontoon and $d$ the draft) and $a / h=1.2$ and $d / h=0.125$ 
240 (referred to be single pontoon 2) are plotted in Fig. 5. Note that the volume of the pontoon of the 241 single case equals to the total volume of the pontoons of the dual pontoon case. It can be seen that 242 the reflection coefficient generally increases with increasing $k h$ but with oscillations, whose 243 amplitude appears to increase with increasing $D / h$. Similar phenomenon can be found for the total 244 CWR $\eta_{\text {total. }}$. The system with a smaller $D / h$ gives a broader frequency range, for which the $K_{\mathrm{T}}<0.5$. 245 For the four cases, the total CWR increases with increasing $k h$ firstly and then decreases after it 246 reaches the maximum. The frequency ranges corresponding to $\eta_{\text {total }}>20 \%$ are similar. In conclusion, 247 the system with a smaller spacing may give a broader effective frequency range in terms of $K_{\mathrm{T}}<0.5$ 248 and $\eta_{\text {total }}>20 \%$. The effective frequency ranges are $1.55<k h<5.77,1.78<k h<5.71,2.02<k h<$ 2495.42 and $1.78<k h<5.67$ and the corresponding effective bandwidths are 4.22, 3.93, 3.40 and 3.89 250 for $D / h=0.2,0.4,0.6$ and 1.0, respectively. The effective bandwidths of an isolated single pontoon 1 251 and an isolated single pontoon 2 are bandwidth 1.09 and 2.53, respectively. It can be seen that 252 effective frequency bandwidth of the two-pontoon system is broader than that of isolated single 253 pontoons.
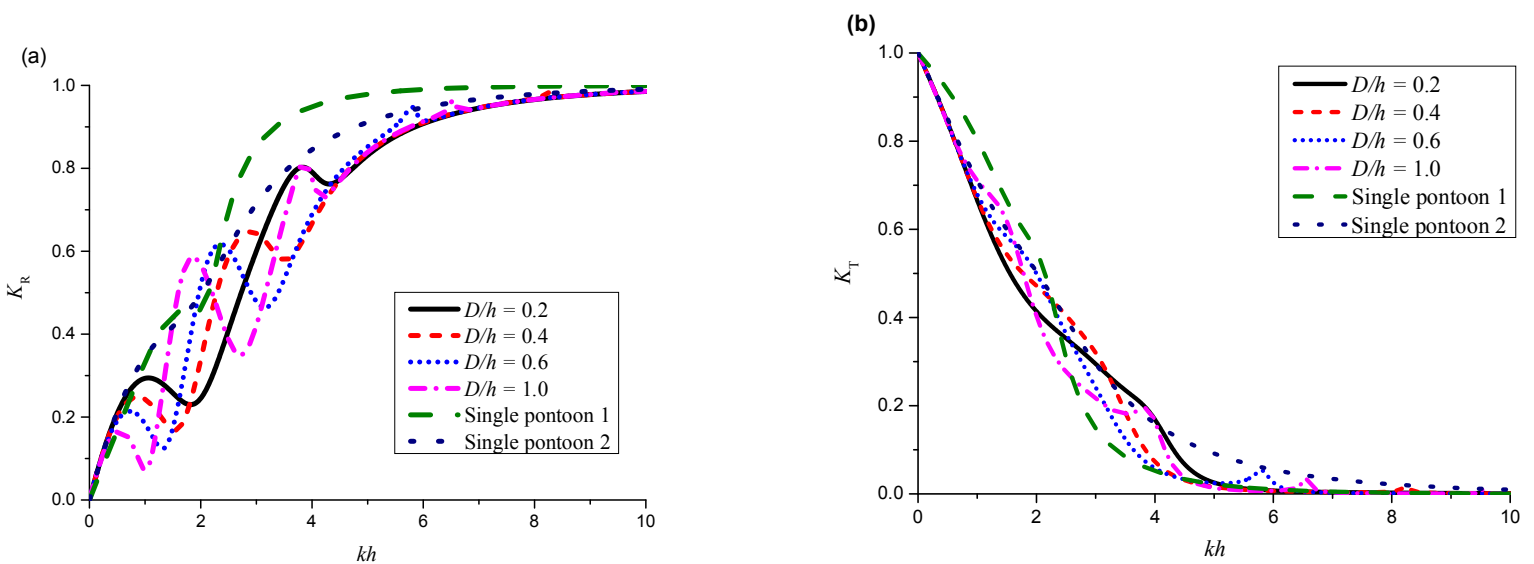


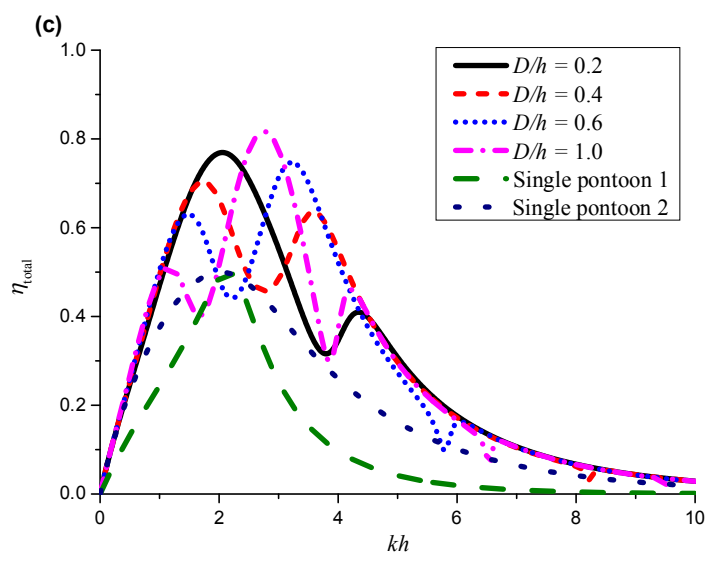

257 Figure 5 Variations of the reflection coefficient $K_{\mathrm{R}}$, transmission coefficient $K_{\mathrm{T}}$ and total capture 258 width ratio $\eta_{\text {total }} v s$ the dimensionless wavenumber $k h$ for cases with different spacings $\left(a_{1} / h=a_{2} / h=\right.$ $\left.2590.6, d_{1} / h=d_{2} / h=0.125\right)$. The size of single pontoon 1 is $a / h=0.6$ and $d / h=0.25$, and the size of 260 single pontoon 2 is $a / h=0.12$ and $d / h=0.125$.

\subsubsection{Effect of the breadth ratio $\left(a_{1} / a_{2}\right)$}

It has been proved that, for a system consisting of two identical pontoons, the natural frequency in the heave mode of each pontoon and the spacing between the two pontoons are the critical factors for the performance of the dual pontoon system. From the point view of the engineering cost, the smaller the total volume of the two pontoons, the lower cost of the integrated system. The cost reduction should not compromise the performance of the system. It is understood that the reduction of the draft or the breadth can lead to a decrease in the volume of a pontoon. Therefore, the system consisting of two non-identical pontoons (i.e., the two pontoons with different dimensions and different natural periods) is of interest. In this section, the effect of breadth ratio of the two pontoons $\left(a_{1} / a_{2}\right)$ is investigated. Two scenarios are considered: (1) $a_{2} / h$ fixed as 0.6 with three breadths of the front pontoon of $a_{1} / h=0.2,0.4$ and 0.6 and (2) $a_{1} / h$ fixed as 0.6 with three breadths of the rear 273 pontoon of $a_{2} / h=0.2,0.4$ and 0.6 . The drafts and the distance are defined as $d_{1} / h=d_{2} / h=0.125$ and $274 D / h=0.2$, respectively. In the discussion, all the results are compared with the results of a reference case of $a_{1} / h=a_{2} / h=0.6, d_{1} / h=d_{2} / h=0.125$ and $D / h=0.2$. 

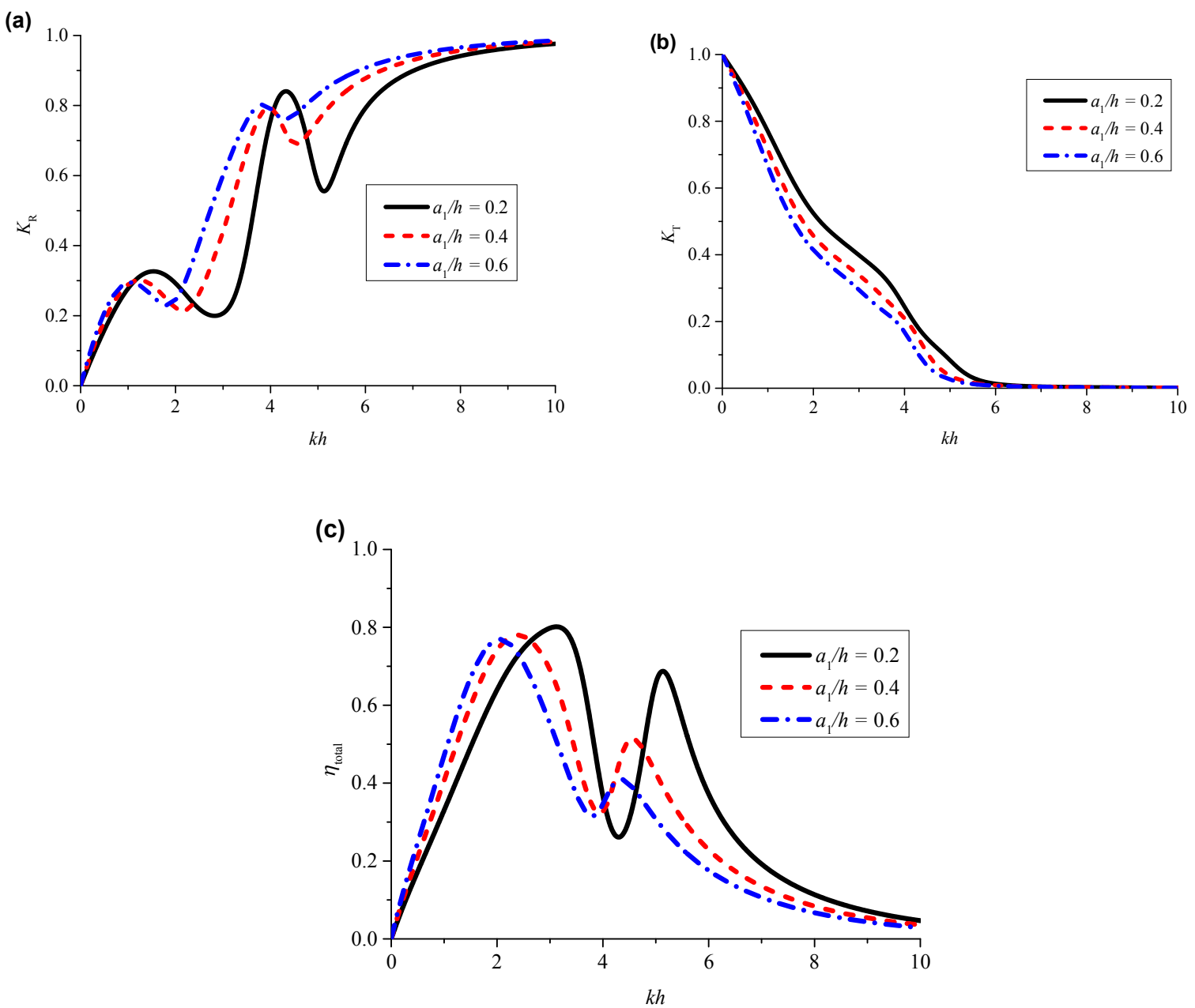

Figure 6 Variations of the reflection coefficient $K_{\mathrm{R}}$, transmission coefficient $K_{\mathrm{T}}$ and total capture width ratio $\eta_{\text {total }} v s$ the dimensionless wavenumber $k h$ for cases with different breadth ratios. $\left(a_{2} / h=\right.$ $\left.0.6, d_{1} / h=d_{2} / h=0.125, D / h=0.2\right)$

Figs. 6(a-c) show the results of the reflection coefficient $K_{\mathrm{R}}$, transmission coefficient $K_{\mathrm{T}}$ and total CWR $\eta_{\text {total }}$ corresponding to the three values of $a_{1} / h(=0.2,0.4$ and 0.6$)$ with a fixed $a_{2} / h=0.6$. It can be seen that the transmission coefficient of the system decreases and the frequency range for $\eta_{\text {total }}>20 \%$ is broadened slightly with the decrease of the $a_{1} / a_{2}$. That is to say, the effective frequency range changes little by comparing with the case of the $a_{1} / h=a_{2} / h=0.6, d_{1} / h=d_{2} / h=$ 0.125 and $D / h=0.2$. The effective frequency ranges are $2.15<k h<6.93,1.77<k h<6.24$ and 1.55 $<k h<5.76$ and the bandwidths are $4.78,4.47$ and 4.21 for $a_{1} / h=0.2,0.4$ and 0.6 , respectively. 
(a)

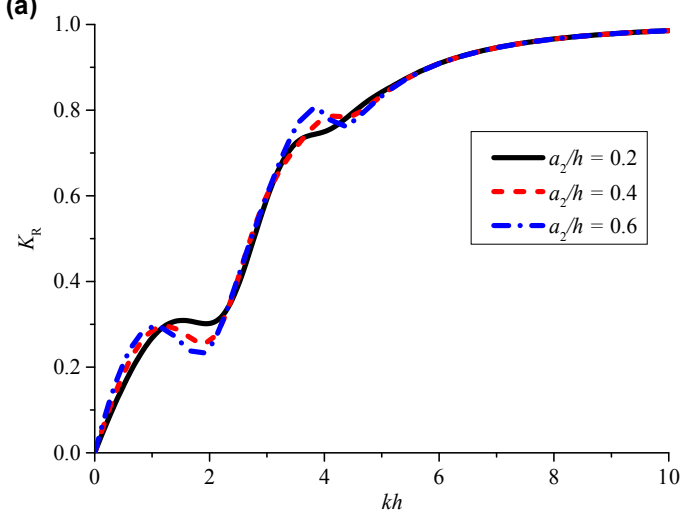

(b)

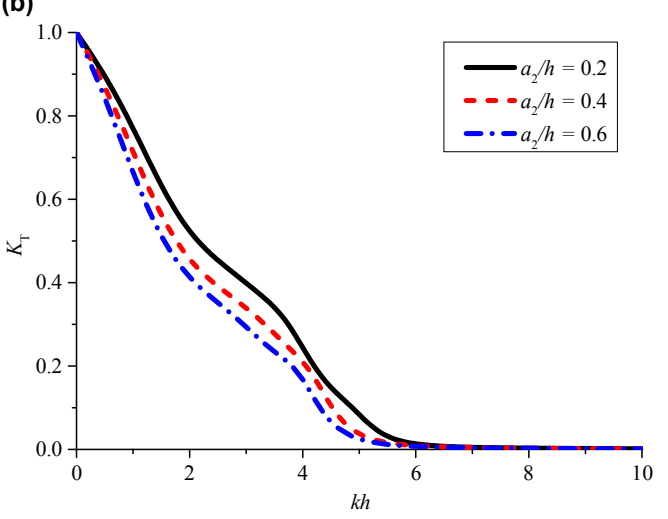

(c)

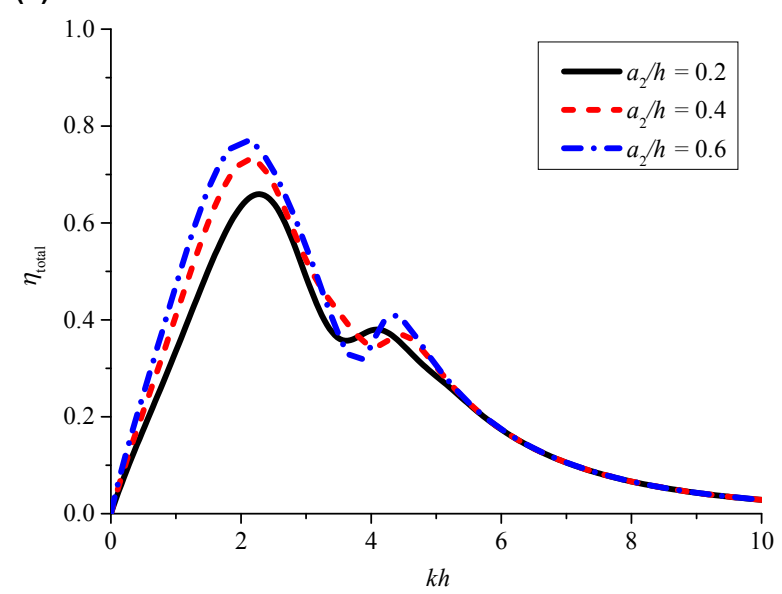

Figs. 7(a-c) show the results corresponding to the three values of $a_{2} / h(=0.2,0.4$ and 0.6$)$ with a fixed $a_{1} / h=0.6$. It can be seen that the variations of $K_{\mathrm{R}} v s k h$ of the three cases are similar to each other. The transmission coefficient becomes smaller with the decrease of the $a_{1} / a_{2}$ at the low frequency region. The effective frequency ranges for $a_{2} / h=0.2,0.4$ and 0.6 are $2.14<k h<5.74$, $1.77<k h<5.74$ and $1.55<k h<5.74$ and the bandwidths are 3.60, 3.97 and 4.19, respectively. The effective frequency bandwidth becomes broader with the decrease of the $a_{1} / a_{2}$. By comparing the results shown in Figs. 6 (b-c), it is found that the configuration with a smaller front pontoon and a bigger rear pontoon has wider effective bandwidth than the opposite arrangement of the pontoons. 


\subsubsection{Effect of the draft ratio $\left(d_{1} / d_{2}\right)$}

The effect of the draft ratios $\left(d_{1} / d_{2}\right)$ is investigated in this section. Firstly, the group with three drafts of $d_{1} / h=0.04,0.08$ and 0.125 are considered. The other parameters are fixed as $d_{2} / h=0.125$, $a_{1} / h=a_{2} / h=0.6$ and $D / h=0.2$. Secondly, performance of the opposite configuration (i.e., $d_{1} / h=$ $0.125 d_{2} / h=0.04,0.08$ and 0.125$)$ is investigated. Similar to Section 3.2.4, the reference case is not changed.

(a)

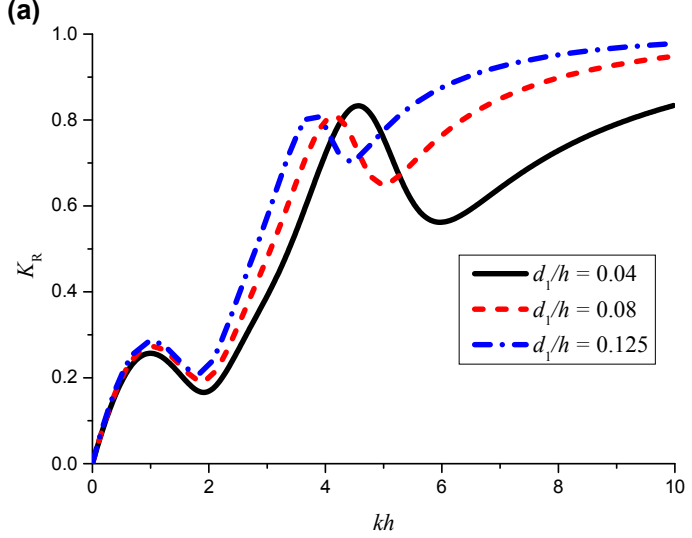

(b)

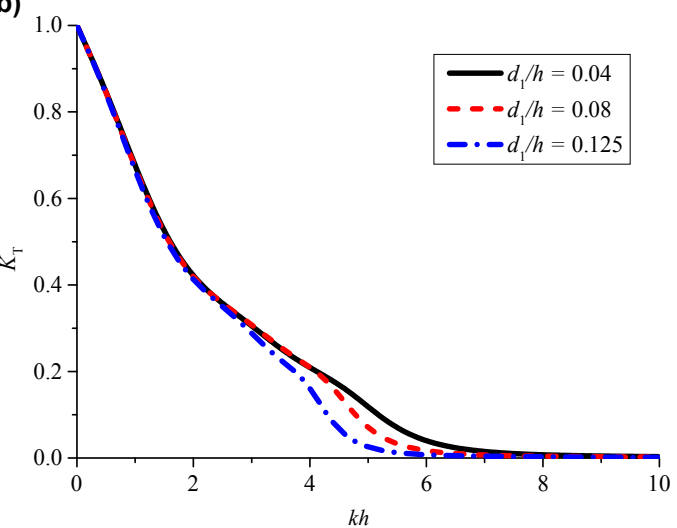

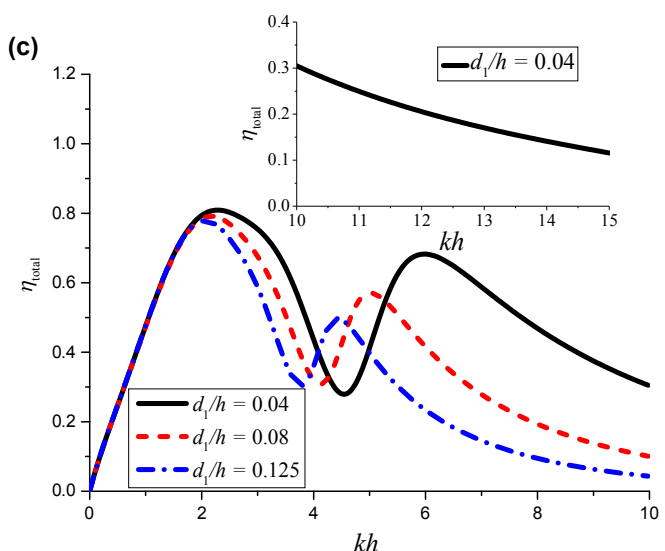

Figure 8 Variations of the reflection coefficient $K_{\mathrm{R}}$, transmission coefficient $K_{\mathrm{T}}$ and total capture width ratio $\eta_{\text {total }}$ for pontoons with different draft ratios $\left(d_{2} / h=0.125, a_{1} / h=a_{2} / h=0.6, D / h=0.2\right)$.

The influence of the draft ratio $\left(d_{1} / d_{2}=0.32,0.64\right.$ and 1$)$ on the reflection coefficient $K_{\mathrm{R}}$, transmission coefficient $K_{\mathrm{T}}$ and total CWR $\eta_{\text {total }}$ is shown in Figs. 8(a-c). It can be seen that the strongest oscillation occurs for the case with the smallest draft ratio. The transmission coefficient is found to be affected by $d_{1} / h$ only at region of $3.5<k h<7.0$. The effective frequency range becomes broader with the decrease of the draft ratio. The $K_{\mathrm{T}}$ values for the three cases are the almost same as 
$k h<2$. That is to say, the rear pontoon with a large size determines the transmission coefficient of the integrated system in long waves. In terms of the total CWR, the integrated system gives a broader effective frequency range. The effective frequency ranges for $K_{\mathrm{T}}<0.5$ and $\eta_{\text {total }}>20 \%$ are $1.60<k h$ $<12.14,1.58<k h<7.9$ and $1.55<k h<6.32$ for $d_{1} / d_{2}=0.32,0.64$ and 1 , respectively. Accordingly, the bandwidths are 10.56, 6.32 and 4.77. In a word, the integrated system with the smaller draft ratio performs better than that of the identical case in terms of the effective frequency bandwidth.

(a)

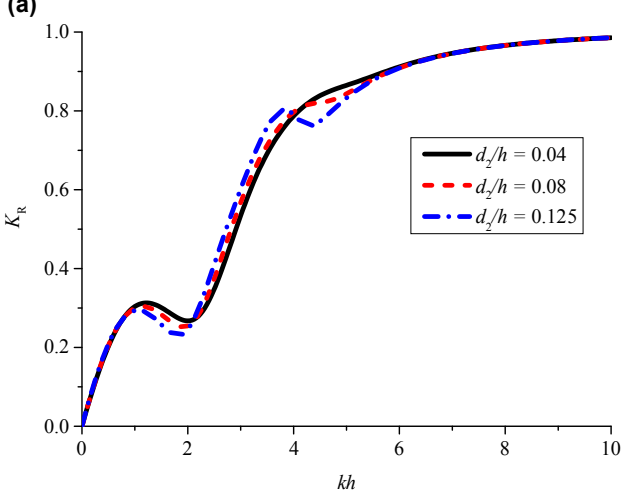

(b)

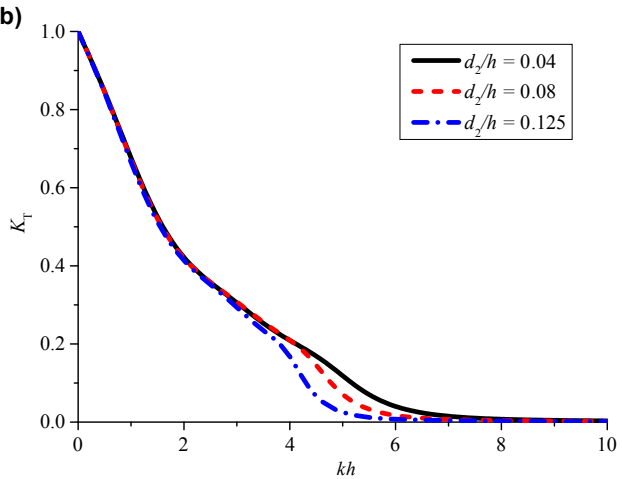

(c)

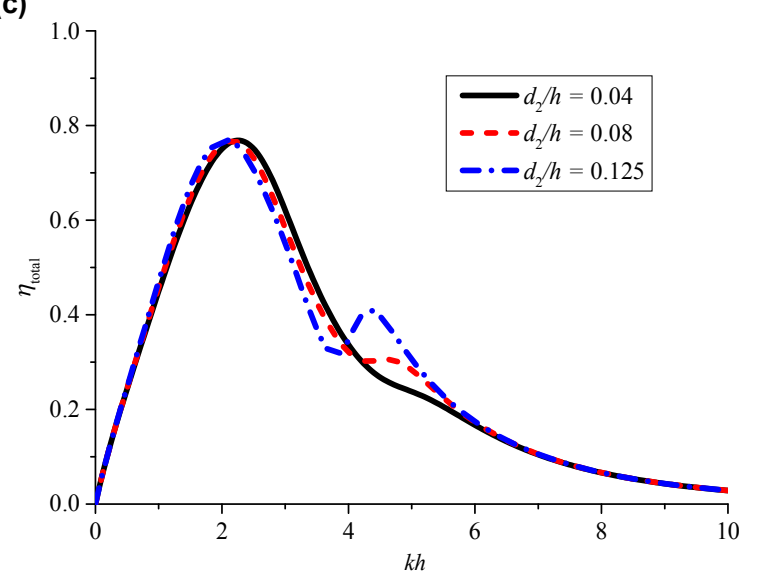

Figure 9 Variations of the reflection coefficient $K_{\mathrm{R}}$, transmission coefficient $K_{\mathrm{T}}$ and total capture width ratio $\eta_{\text {total }}$ for pontoons with different draft ratios $\left(d_{1} / h=0.125, a_{1} / h=a_{2} / h=0.6, D / h=0.2\right)$.

Figs. 9 (a-c) shows the results as the larger pontoon is located at the upstream side. The effective ranges are $1.60<k h<5.74,1.58<k h<5.73$ and $1.55<k h<5.57$ for $d_{2} / h=0.04,0.08$ and 0.125, respectively. Accordingly, effective frequency bandwidths are 4.14, 4.15 and 4.02, respectively. It can be seen that the bandwidth changes little by decreasing the draft of the rear 
pontoon. By comparing the results shown in Figs. 8 and 9, the configuration with the small-draft front pontoon and large-draft rear pontoon performs better than the opposite configuration in terms of the effective frequency bandwidth. But the transmission coefficient changes very little for the two configurations with the same total volume.

\subsection{Discussions}

The hydrodynamic performance of an integrated breakwater system with two floating pontoons is investigated. Results showed that the two-pontoon system results in a broader effective frequency range in terms of the qualified transmission coefficient and acceptable total CWR than a single-pontoon system. It is understood that the volume of the floating structures affects its cost. Now we take the case with parameters of $d_{1} / h=0.04, d_{2} / h=0.125, a_{1} / h=a_{2} / h=0.6$ and $D / h=0.2$ as an example, the total volume of two pontoons is much smaller than that of the single pontoon with the parameters of $a / h=0.6, d / h=0.25$ and $a / h=1.2, d / h=0.125$. Therefore, the two-pontoon system is much more acceptable economically.

The comparisons of the performance with different configuration of two non-identical pontoons are conducted. Interestingly, the configuration with a smaller front pontoon and a larger rear pontoon performs better than the opposite configuration in terms of the effective frequency bandwidth. This is because stronger reflection occurs at the high frequency region if the larger pontoon is in front of the smaller pontoon, resulting in lower CWR. Thus, the effective frequency bandwidth is narrower. From the point view of effective frequency bandwidth, the configuration with the smaller front pontoon and the larger rear pontoon is suggested.

It is worthy to note that, since the viscous effect is not considered, the transmission coefficient and the total CWR of the integrated system may be overestimated by using the potential flow theory in the frequency domain. The future work will focus on the physical experiments by adopting the general generator.

\section{Conclusions}

The hydrodynamic properties of a two-pontoon WEC-type breakwater have been investigated 
analytically under the frame of linear potential flow theory. The matching eigen-function method is used to solve the diffraction and radiation problems. The absorbed power is calculated by using the linear PTO damping method. The numerical results for a range of configurations are presented to illustrate the influence of the different wave and structural parameters on the performance of the integrated system. The conclusions are summarized as follows.

(1) The reflection coefficient, transmission coefficient and the total CWR of a system with two identical pontoons strongly depends on the natural frequency in heave mode and the spacing between them;

(2) For a system with two non-identical pontoons but the total volume fixed, the broader effective frequency bandwidth $\left(K_{\mathrm{T}}<0.5\right.$ and $\left.\eta_{\text {total }}>20 \%\right)$ can be achieved for configuration with a front pontoon with small-draft and a rear pontoon with large-draft;

(3) By comparing the transmission coefficient and the CWR of the system with those by the single pontoon, the system with two small pontoons (i.e., the total volume of the two small pontoons is less than that of the single pontoon) can give a better performance.

\section{Acknowledgements}

The authors would like to acknowledge the financial support the National Natural Science Foundation of China (Grant Nos. 51379037, 51679036 and 51628901) and High-Tech Ship Research Projects Sponsored by the Ministry of Industry and Information Technology (MIIT) of China (Grant No. KY10100160023-004). Thanks also go to Dr. Si-Ming Zheng from Tsinghua University for valuable discussions.

\section{References}

Arena, F., Romolo, A., Malara, G., Ascanelli, A., 2013. On design and building of a U-OWC wave energy converter in the Mediterranean Sea: A case study. Paper presented at the ASME 2013 International Conference on Ocean, Offshore and Arctic Engineering.

Babarit, A., 2015. A database of capture width ratio of wave energy converters. Renewable Energy, $80,610-628$.

Babarit, A., Hals, J., Muliawan, M. J., Kurniawan, A., Moan, T., Krokstad, J., 2012. Numerical benchmarking study of a selection of wave energy converters, Renewable Energy, 41(0), 44-63. 
Boccotti, P., 2007. Comparison between a U-OWC and a conventional OWC. Ocean Engineering, 34(5), 799-805.

Chen, B., Ning, D. Z., Liu, C. Q., Greated, C. A., Kang, H. G., 2016. Wave energy extraction by horizontal floating cylinders perpendicular to wave propagation. Ocean Engineering, 121, $112-122$.

Falcão, A. F. d. O., 2010. Wave energy utilization: A review of the technologies. Renewable and Sustainable Energy Reviews, 14(3), 899-918.

Falnes, J., 2002. Ocean waves and oscillating systems: Cambridge University Press.

Ferro, B. D., 2006. Wave and tidal energy: Its Emergence and the Challenges it Faces. Refocus, 7(3), 46-48.

Garnaud, X., Mei, C. C., 2009. Wave-power extraction by a compact array of buoys. Journal of Fluid Mechanics, 635: 389-413.

He, F., Huang, Z. H., 2014. Hydrodynamic performance of pile-supported OWC-type structures as breakwaters: An experimental study. Ocean Engineering, 88(5), 618-626.

He, F., Huang, Z. H., Law, W. K. A, 2013. An experimental study of a floating breakwater with asymmetric pneumatic chambers for wave energy extraction. Applied Energy, 106(11), 222-231.

He, F., Huang, Z. H., Law, W. K. A., 2012. Hydrodynamic performance of a rectangular floating breakwater with and without pneumatic chambers: An experimental study. Ocean Engineering, 51, $16-27$.

He, F., Li, M., Huang, Z. H., 2016. An experimental study of pile-supported OWC-type breakwaters: energy extraction and vortex-induced energy loss. Energies, 9.

Koutandos, E., Prinos, P., Gironella, X., 2005. Floating breakwaters under regular and irregular wave forcing: reflection and transmission characteristics. Journal of Hydraulic Research, 43(2), 174-188.

Li, Y. C., Teng B., 2015.Wave Action on Maritime Structures[M]. Beijing: China Ocean Press.

Martinelli, L., Ruol, P., Favaretto, C., 2016. Hybrid structure combining a wave energy converter and a floating breakwater. Paper presented at the Proceedings of the International Offshore and Polar Engineering Conference.

McCartney, B. L., 1985. Floating breakwater design. Journal of Waterway, Port, Coastal, and Ocean Engineering, 111(2): 304-318.

Mendoza, E., Silva, R., Zanuttigh, B., Angelelli, E., Lykke Andersen, T., Martinelli, L., Nørgaardd 
JQH, Ruol, P., 2014. Beach response to wave energy converter farms acting as coastal defence. Coastal Engineering, 87, 97-111.

Michailides, C., Angelides, D. C., 2012. Modeling of energy extraction and behavior of a Flexible Floating Breakwater. Applied Ocean Research, 35(1), 77-94.

Ning, D. Z., Zhao, X. L, Kang, H. G., Goteman, M., 2016. Hydrodynamic performance of a pilerestrained WEC-type floating breakwater: An experimental study. Renewable Energy, 95:531-541.

Liu, Y, Li., H. J., 2014. A new semi-analytical solution for gap resonance between twin rectangular boxes. Proceedings of the Institution of Mechanical Engineers, Part M: Journal of Engineering for the Maritime Environment, 228(1), 3-16.

Orer, G., Ozdamar, A., 2007. An experimental study on the efficiency of the submerged plate wave energy converter. Renewable Energy, 32(8), 1317-1327.

Takahashi, S., Nakada, H., Ohneda, H., Shikamori, M., 1992. Wave power conversion by a prototype wave power extracting caisson in Sakata port. Paper presented at the International Conference on Coastal Engineering (ICCE).

Vicinanza, D., Contestabile, P., Nørgaard, J. Q. H., Andersen, T.L., 2014. Innovative rubble mound breakwaters for overtopping wave energy conversion. Coastal Engineering, 88(3), 157-170.

Weng, W. K., Chou, C. R., 2007. Analysis of responses of floating dual pontoon structure. China Ocean Engineering, 21(1), 91-104.

Williams, A. N., Abul-Azm, A. G., 1997. Dual pontoon floating breakwater. Ocean Engineering, $24(5), 465-478$.

Williams, A. N., Lee, H. S., Huang, Z. H., 2000. Floating pontoon breakwaters. Ocean Engineering, 27(3), 221-240.

Wolgamot, H. A., Taylor, R. E., Taylor, P. H., 2016. Effects of second-order hydrodynamics on the efficiency of a wave energy array. International Journal of Marine Energy. 15, 85-99.

Zheng, S. M., Zhang, Y. L., 2016. Wave diffraction and radiation by multiple rectangular floaters. Journal of Hydraulic Research, 54(1), 102-115.

Zanuttigh, B., Angelelli, E., 2013. Experimental investigation of floating wave energy converters for coastal protection purpose. Coastal Engineering, 80(4), 148-159. 